\title{
Intraoperative ultrasound in liver and pancreatic surgery
}

\author{
Adrian Bartoș ${ }^{1,2}$, Ioana Iancu ${ }^{1}$, Lidia Ciobanu ${ }^{1,2}$, Radu Badea ${ }^{1,2}$, Zeno Spârchez ${ }^{1,2}$, Dana \\ Monica Bartoș 1,2
}

1"Prof Dr Octavian Fodor" Regional Institute of Gastroenterology and Hepatology, ${ }^{2}$ Iuliu Haţieganu University of Medicine and Pharmacy, Cluj-Napoca, Romania

\begin{abstract}
This paper summarizes the current knowledge of intraoperative ultrasonography (IOUS) in open and laparoscopic abdominal surgery. The abdominal IOUS contributes to the diagnosis and staging (for parenchymal organ tumors), to establish surgical procedure and to guide surgical maneuvers. The main applications are represented by liver, biliary tract and pancreatic pathology. Diagnostic approaches are frequently combined with therapeutic purposes. The technique, equipment, training, benefits and limits of IOUS in abdominal surgery are discussed and cases from our experience are used as examples.

Keywords: intraoperative ultrasound; laparoscopic ultrasound; contrast-enhanced ultrasound; hepatocellular carcinoma; liver metastasis; pancreatic tumor
\end{abstract}

\section{Introduction}

Intraoperative ultrasound (IOUS) was used in clinical practice for the first time in the 60s using the A mode to identify stones in the kidney [1] and in the common bile duct [2]. The development of the real-time B mode and adapted transducers, was accredited to Sigel et al [3] in 1980 , during biliary surgery, to identify the common bile duct and stones in inflammatory condition, using a sterile high-resolution transducer (7.5 MHz). In 1982 Fukuda et al [4] described the echolaparoscopy for liver screening and for gastrointestinal cancer staging. Ten years later, in 1991, Jakimowiczet al [5] reported the preliminary experience for laparoscopic ultrasound (LUS) for screening the biliary tract during laparoscopic cholecystectomy.

Received 02.10.2020 Accepted 09.11.2020

Med Ultrason

2021, Vol. 23, No 3, 319-328

Corresponding author: Ioana Iancu

"Prof Dr Octavian Fodor" Regional Institute

of Gastroenterology and Hepatology

19-21 Croitorilor Street,

400162, Cluj, Romania

E-mail: iancuioana2011@gmail.com
Intraoperative ultrasound during open access or laparoscopic approach share the same principle: the direct contact of the transducer with the screened organ. For LUS, some targets are more difficult to be localized.

The technical achievements permitted the extension of IOUS in many fields of surgery: nervous system, cardiovascular system, endocrine gland, breast [6-9]. The benefits of IOUS has expended beyond diagnostics; the ultrasound image represents nowadays, a guidance for surgical maneuvers. The new ultrasound techniques, such as Doppler mode, contrast-enhanced ultrasound (CEUS) or elastography, are frequently used during IOUS both for diagnostic and therapeutic purposes.

However, the use of IOUS is not wide spread in real-life practice; the possible explanations might be the lack of specialized training for surgeons and the development of non-invasive imaging techniques such as the computed tomography (CT) scan and magnetic resonance imaging (MRI).

The aim of our work was to review using a narrative approach the main IOUS applications in abdominal pathology, emphasizing their role in the diagnosis and therapeutics, in comparison with other imaging techniques. 


\section{Equipment and techniques}

The scanner used in the intra-operative room should be a compact, easy-to-operate, mobile system that provides high quality images [10]. An ideal ultrasound device used for intraoperative examinations must ensure the possibility of performing CEUS and elastography, besides grey scale, pulsed Doppler and color/power Doppler. These devices must be ergonomic, of small size and must allow periodic cleaning and sterilization.

Although IOUS can be performed using conventional transducers, for interventional maneuvers, it is necessary to use dedicated transducers which are easily handled in narrow anatomical regions (the inter-hepato-phrenic space or the lesser sac) because of their small size and ergonomic design (fig 1). Different types of transducers are available (linear array, convex linear array and phased array or sector) [10]. An ideal transducer for intraoperative purposes must generate high frequencies (7-13 MHz) with low penetration (up to $12 \mathrm{~cm}$ ) [10]. By applying the transducer directly on the targeted organ, the artefacts due to signal reduction disappear. The wide scanning window is also mandatory and can also be obtained for linear probes by using the „trapezoidal window" feature. For IOUS J- or T-shape transducer are used. LUS uses dedicated transducers with an angulating tip, which allows up and down movements of $90^{\circ}$ [10] (fig 2). Intraoperative use assumes compliance with the rules of asepsis, which is why transducers should allow sterilization. Of course, this can also be easily ensured by using sterile covers such as in laparoscopic surgery.

The technique for exploratory procedure for IOUS and LUS is based on the principle of direct contact between the probe and the target area. A complete evaluation of the target area should be performed systematically and thoroughly screened by multiplanar scanning maneuvers.

For US guided surgical resection of malignant tumors, detailed vascular structures and networks are detected with color Doppler mode or CEUS, in standardized multiplanar sections. For example, in modern hepatic surgery, for both hepatocellular carcinoma and colorectal liver metastases, the IOUS allows so-called "radical but conservative surgery". This is based on the recognition of the relationship between liver lesions and intrahepatic bilio-vascular structures. Knowing this data, the surgeon can guide his resection line, respecting the Glisson pedicles, and suprahepatic veins, with the ultimate goal of preserving as much functional hepatic parenchyma as possible [11-15]. For anatomical resection of the liver, the compression of segmental portal branches is done between the transducer and the operator's fingers, resulting

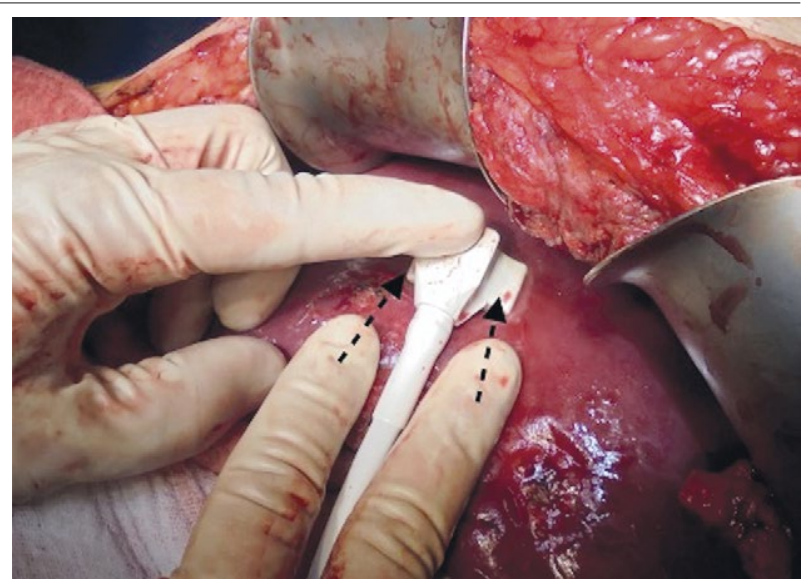

Fig 1. Handling a dedicated IOUS transducer in the interhepatophrenic space (miniconvex transducer, 1-13 MHz, 65을 Hitachi Aloka Medical Ltd / Japan)

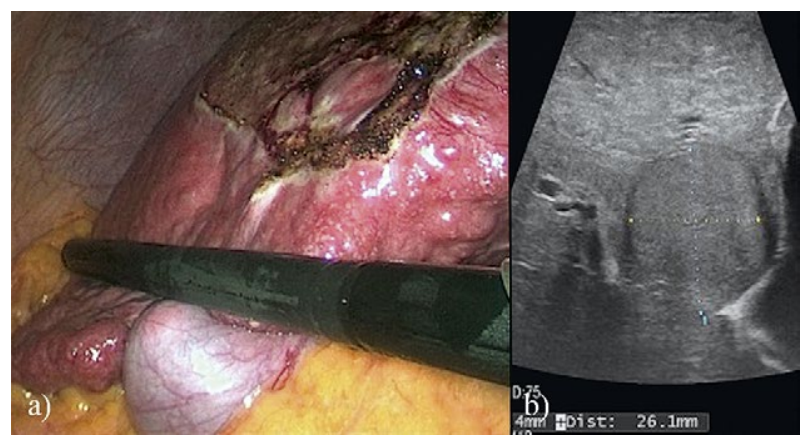

Fig 2. Laparoscopic IOUS of the liver performed by using a transducer dedicated for this approach (laparoscopic transducer, 13-2 MHz, 36 mm. Hitachi Aloka Medical Ltd / Japan): a) intraoperative aspect; b) ultrasound aspect (hepatocarcinoma nodule, tangent to the wall of the gallbladder)

in a transient ischemia of the targeted parenchyma, which can be marked with the electrocautery; then the resection is made along the demarcation line [15-21].

IOUS guided procedures (such as biopsy or ablative techniques) are done for subcapsular lesions located in the immediate vicinity of important structures (diaphragm, stomach and gallbladder) or for difficult to approach tumors (caudal lobe). It might be performed through open surgery or by laparoscopic approach, which is preferred. Ultrasound has in this context two major roles: first to guide the tools and the second to confirm the ablative treatment efficacy mostly by using contrast-enhanced agents [22-25].

Ultrasound characterization of superficial lesions (hepatic or pancreatic subcapsular lesions) is often difficult, especially in cases of irregular visceral surfaces (liver cirrhosis, chronic pancreatitis) due to artefacts caused by the imperfect contact between the transducer and the surface of the organ. This can be exceeded by interposing 


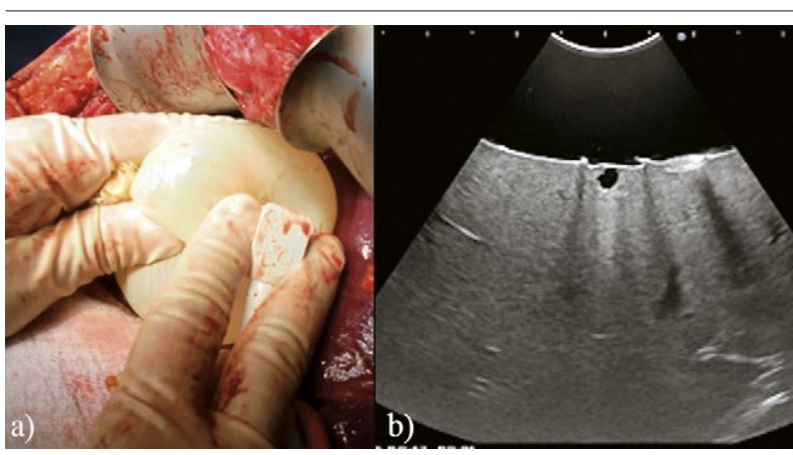

Fig 3. The examination of superficial liver lesions during IOUS: a) interpolating of a saline solution medium between the transducer and the targeted lesion; b) high resolution obtained for a superficial lesion (cyst)

a liquid media between the transducer and the targeted organ (fig 3).

From a technical point of view, IOUS is different from conventional ultrasound because the scanning of the targeted organs is performed directly and locating different lesions often requires handling into narrow and less accessible anatomical regions (interhepatophrenic space and lesser sac). It is often necessary to mobilize these organs and create specific access paths, such as sectioning the ligaments of the liver, sectioning of the gastro-colic ligament, performing the Kocher maneuver. Also, the standardization of conventional ultrasound sections and plans does not overlap completely in IOUS, as there are many situations in which it is necessary to scan from the posterior surface of the organ (for example: the visceral surface of the liver).

IOUS is an operator-dependent technique. The examination can be performed by physicians specialized in imaging diagnosis, but the most benefits could be obtained from surgeons by transforming ultrasound into an „extension" of the hands, under the conditions of the routine use in the operating room. For therapeutical purposes the learning curve is considered to be completed after 40 examinations of the pancreas and 50 of the liver [26]. For surgeons, this can be obtained only after obtaining the competence in general ultrasound, basic levels of knowledge being mandatory for obtaining optimum results.

\section{Indications}

\section{Liver pathology}

Benign pathology

For benign pathology IOUS is useful in cases of multiple lesions (polycystic liver, abscesses or multiple hydatid cysts), complex morphology (multicameral/poliseptate cysts) or topography difficult to assess by inspection/palpation (deep lesions). In these situations, ultrasound makes its contribution by shortening the surgical time (rapid finding of lesions) and providing complete treatment with minimal recurrence risk. Minimally invasive (laparoscopic) treatment is facilitated by the association of IOUS which replace the palpation used in „classic" surgery. For the complex situation where the hand-assisted laparoscopic technique is used to maintain the minimally invasive character of the procedure, the IOUS might be essential (fig 4). The choice of optimal surgical treatment can be influenced by IOUS, an example being represented by hydatid cysts: partial pericystectomy versus ideal cystectomy, a decision that is taken according to the topography and the proximity of vascular structures, aspects that can be fully appreciated by IOUS.

Recently, contrast-enhanced IOUS (CE-IOUS), IOUS elastography, and IOUS-guided hepatic surgery have attracted increasing interest and are expected to lead to the broader implementation of IOUS.

\section{Hepatocellular carcinoma}

In cirrhotic livers, the differential diagnosis between regenerative nodules and hepatocellular carcinoma (HCC) can be challenging due to fibrosis involvement.

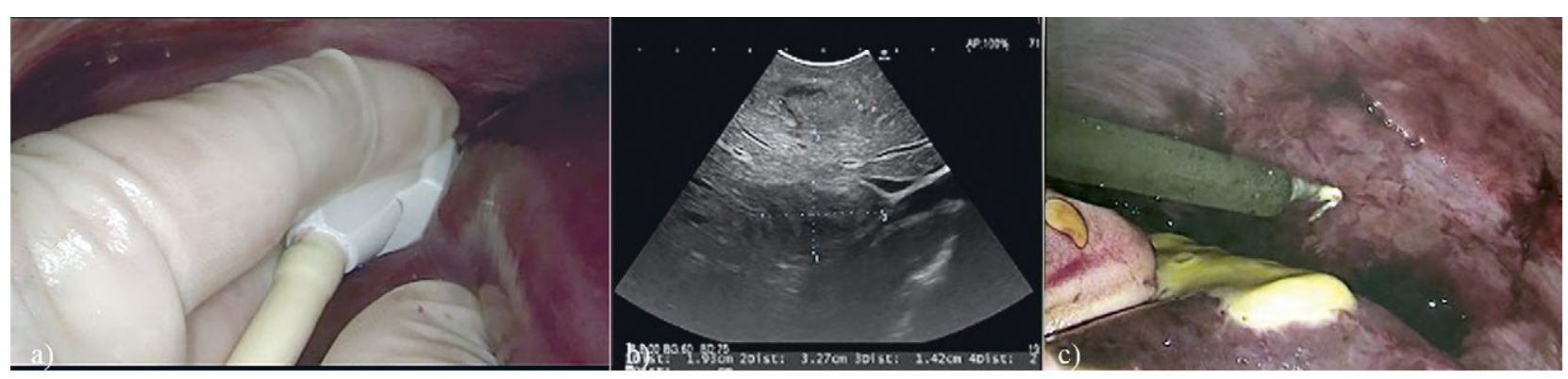

Fig 4. Evacuating a hepatic abscess in a patient with Crohn disease and terminal ileitis. Because the right hemicolectomy was performed laparoscopically, the drainage was performed under ultrasound guidance in order to maintain the minimally invasive character of the procedure: a) intraoperative aspect, with the transducer positioned in the interhepatophrenic space by a hand assisted approach (minilaparothomy), under laparoscopic guidance; b) ultrasound aspect with the liver abscesses, before evacuation; c) intraoperative aspect with the evacuation of the abscess 
The advantage of IOUS is the possibility to better characterize these lesions, nowadays based on the vascular pattern in CEUS and to apply the best surgical treatment: resection or intraoperative ablation. Both the resection and the ablative techniques might be guided by ultrasound, in order to preserve the non-tumoral liver parenchyma. The treatment efficacy assessment can be done "on-site" with the help of contrast-agents.

Early reports emphasized the IOUS role for the detection of new HCC nodules and subsequently the changes in therapeutic approaches in $1 / 3$ of the patients [27,28]. The CE-IOUS is able to depict the vascular pattern of the cirrhotic nodules and to accurate differentiate between regenerative nodules and hepatocellular carcinoma [29]. Contrast enhanced IOUS (CE-IOUS) details the microcirculation perfusion of HCCs more sensitively than preoperative CEUS [29].

The typical vasculature features of CE-IOUS for $\mathrm{HCC}$ on a cirrhotic liver are represented by early contrast enhancement in the arterial phase with fast washout in the venous phase [30,31]. The most frequent used contrast agent is SonoVue (Bracco, Italy). Another vascular pattern corresponding to poorly differentiated HCC is characterized by a hypoechoic pattern with a basket-like peripheral arterial neovascularization [32].

The advantage of this intraoperative staging is the possibility of real-time modification of the surgical therapy decision and expansion of the resection or intraoperative ablation.

\section{Liver metastasis}

Regarding liver metastases after colorectal cancer, evidence shows that $10-40 \%$ of these are not accessible to palpation during surgery [33]. Literature data documented a high accuracy for IOUS for liver metastasis de-

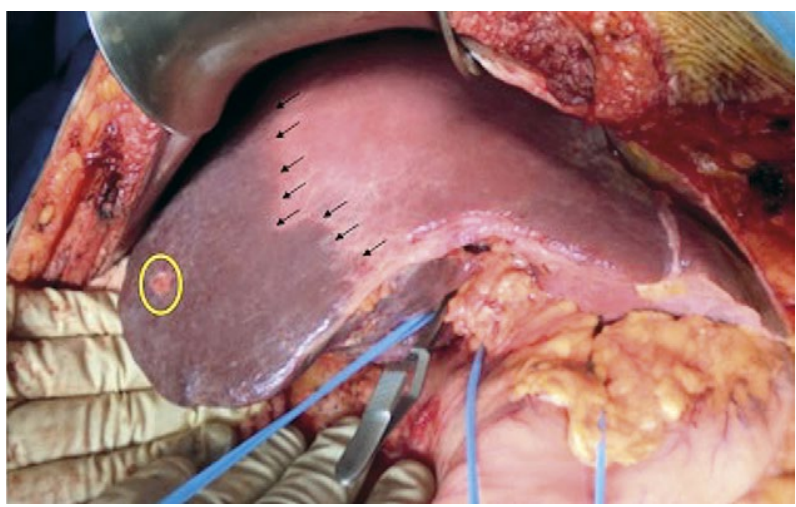

Fig 5. Ischemic delimitation of the future liver's resection line (real anatomic resection) after ultrasound guided extraglissonian clamping of the vascular pedicle for segments VI and VII (geographic map aspect). Surgical indication for multiple hepatic metastases, located in liver segments VI and VII. tection $(99 \%)$ [34]. IOUS changed the operative decision in up to $20 \%$ of cases [33]. Moreover, the association of CE-IOUS can increase diagnostic accuracy, modifying surgical conduct in more than one third of cases $[35,36]$.

IOUS and, more recently, CE-IOUS proved to have a higher accuracy diagnostic value than preoperative imaging (CT, MRI) [36]. The present international guidelines recommend CE-IOUS to be routinely performed in all patients with hepatic metastases proposed for liver resections [37].

A special situation is represented by the hepatic metastases receiving chemotherapy. The complete response, with the actual disappearance of the lesions, is present in only two thirds of the cases [38]. In the remaining one third, the false imaging response is due to the effect of the chemotherapy on the echogenic metastasis which become isoechoic. Thus, CE-IOUS has a crucial role for these patients, being the only examination able to detect the vascular patterns of the "disappearing" nodules and can establish the final diagnosis [39]. By merging the images obtained prior to chemotherapy (by CT or MRI) with real time ultrasound images (Real-time Virtual Sonography - RVS), the detection of the regions of interest, which cannot be visualised because of the isoechoic character mentioned above, might be possible.

Nowadays the role of real-time elastography during IOUS is increasing; it can increase the degree of lesion detection by $8 \%$, having the potential to differentiate between malignant and benign $[40,41]$.

\section{Guiding liver resection}

Patients diagnosed with HCC or with multiple grouped hepatic metastases can benefit from an anatomical resection, guided by ultrasound, which ensures a radical resection of the targeted lesions. In this way,
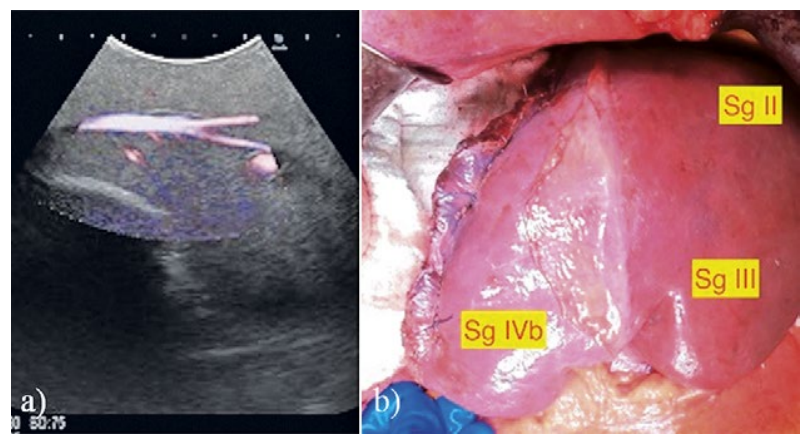

Fig 6. Intraoperative identification of medium suprahepatic vein (longitudinal - color Doppler IOUS) (a) and the guided hepatic resection, tangent to the medium suprahepatic vein, in order to preserve the hepatic parenchyma (segment IVb) (bafter resection), for an intrahepatic cholangiocarcinoma of segment VIII extended to segment IVa, in a patient presenting with chronic hepatopathy and low functional reserve. 
a maximum oncological result is achieved, by minimizing the risk of leaving in situ synchronous satellite micro metastases [42]. Another advantage of the anatomical resections is lower postoperative morbidity by minimizing the risk of bleeding from the resection line or necrosis through a devascularized remnant tissue $[18,21]$. The technical attainment of these resections involves the ultrasound identification of the vascular pedicle of the targeted liver segment, its later clamping (intraparenchymatous or extraglissonian) or its digital compression between the transducer and the surgeon's finger [20]. A clear demarcation of the resection line is obtained by ischemic delimitation of the parenchyma (fig 5).

The utility of IOUS is also maximal for "radical but conservative resections", a term introduced by Torzilli et al [43], with applicability in patients with multiple hepatic masses disseminated in both lobes or patients with chronic hepatopathy, low functional reserve and indication of liver resection (HCC, cholangiocarcinoma, liver metastases). This technique involves the ultrasound identification of lesions and surrounding vascular pedicles, with the establishment of a radical surgical strategy with appropriate oncological resection margins, but with the maintenance of as much as possible viable and functional hepatic parenchyma (fig 6).

Transparietal or intraoperative ablative techniques have well-established indications in the literature [44], ultrasound being mandatory when such a surgical maneuver is required. Intraoperative ablation through laparotomy is usually performed after the liver is mobilized; the ultrasound-guided insertion of the radiofrequency (RFA) or microwave (MWA) needle is facilitated by positioning the transducer directly on the surface of the liver (fig 7). Special scenarios are represented by a laparoscopic approach in cirrhotic patients with coagulation disorders, where a percutaneous eco-guided approach is not possible (subcapsular tumors located in the proximity of other organs - gallbladder, diaphragm, stomach) or in patients with advanced neoplasms in which the treatment of liver metastases is desired to be performed concurrently with the resection of the primary tumor or as a preparation for neoadjuvant treatment $[45,46]$. Laparoscopic approach for liver ablation is possible only with laparoscopic IOUS (L-IOUS), which enables the insertion of the ablation needle under direct ultrasound guidance (fig 8). From our experience, the laparoscopic technique brings the benefits of the minimally invasive approach in selected cases [47]. Also, general anesthesia can be replaced with chest epidural anesthesia. Irrespective of the approach, the post-ablative site check can be performed intraoperatively by using CE-IOUS (fig 9). If the CEIOUS detects remaining neoplastic tissue, the ablation

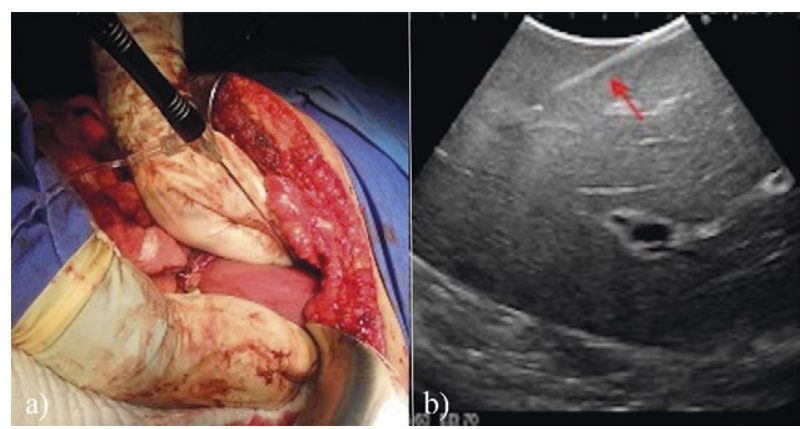

Fig 7. Intraoperative ablation of a hepatic lesion, under ultrasound guidance: a) intraoperative aspect with the RFA electrode; b) IOUS aspect with the guidance of the RFA electrode (arrow).

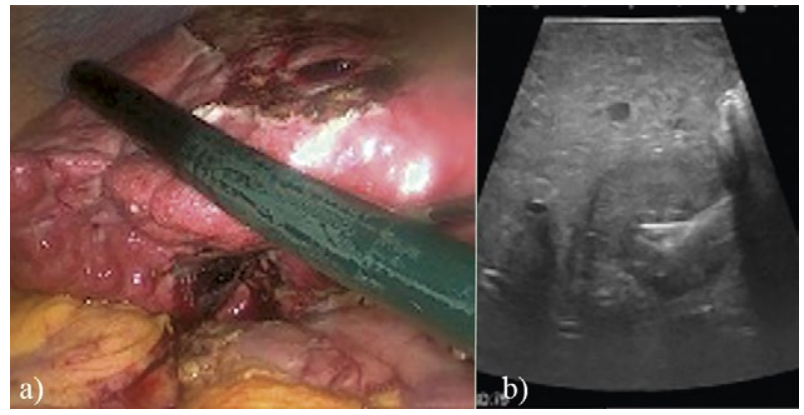

Fig 8. Laparoscopic ablation of hepatic tumors (HCC): a) intraoperative aspect with the laparoscopic transducer and the ablation needle introduced under ultrasound guidance; b) IOUS aspect with the RFA needle opened into the tumor (Strarburst ${ }^{\circledR}$ ).

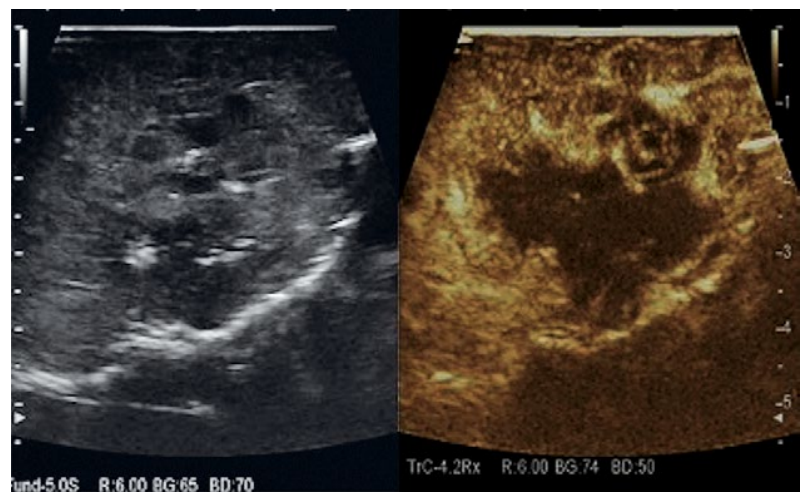

Fig 9. CE-IOUS (SonoVue ${ }^{\circledR}$ ) of a hepatic tumor (HCC) located in segment III, after laparoscopic RFA; no contrast intake of the ablation site in arterial, portal and late time.

session can be repeated in the same operative session, in order to complete the necrosis area.

\section{Pancreatic pathology}

Less commonly used, IOUS might have important applications in both malignant and benign pancreatic pathology [48].

IOUS might be useful in polycystic pancreatic disease, especially when minimally invasive treatment is 


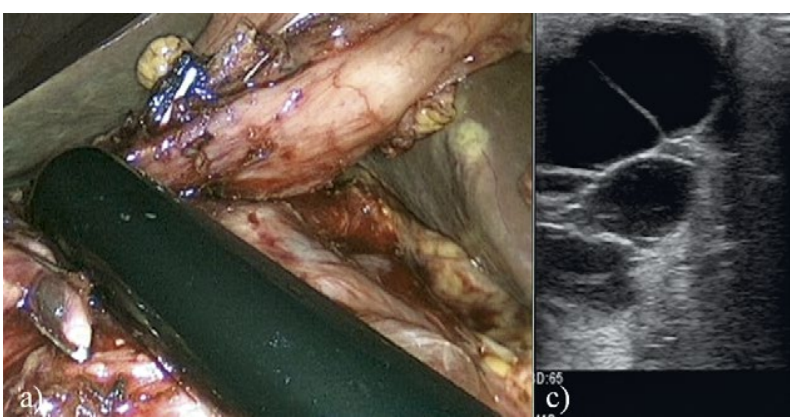

Fig 10. Pancreatic IOUS in a patient with polycystic pathology, with obstructive jaundice following the compression of the common bile duct by a cyst located in the head of the pancreas: a) intraoperative aspect with the laparoscopic transducer, after entering the lesser sac; b) IOUS highlighting of the cyst located in the head of the pancreas with compression of the common bile duct $(*)$, prior to laparoscopic pickling.

desired (laparoscopic approach). Although rare, cases with Von Hippel Lindau syndrome with cysts in the head of the pancreas which compress the common bile duct can benefit from a laparoscopic approach. By performing this procedure, guided by ultrasound, the risk of damaging important surrounding structures (Wirsung duct, peripancreatic vascular arcades) is minimized (fig 10).

Chronic pancreatitis might benefit from surgery in cases of obstructive lithiasis of Wirsung's duct (when endoscopic treatment is not possible). IOUS facilitates the rapid detection of the main pancreatic duct prior to its longitudinal incision in order to perform a pancreatico-jejunostomy (fig 11). The use of CE-IOUS might help the differential diagnosis of a pancreatic pseudotumor; the lack of a focal vascular pattern suggestive of malignancy corroborated with the extemporaneous histopathology exclude adenocarcinoma (fig 12).

The internal drainage of pseudocysts developed after acute pancreatitis is indicated by: more than $6 \mathrm{~cm}$ in diameter, the presence of a mass effect (compression), intracystic hemorrhage and superinfection of the cyst [49]. In addition to endoscopic and „classic” surgical treatment, the pseudocystic-gastro-anastomosis performed laparoscopically by transgastric approach can be an efficient minimally invasive solution which provides a wide communication between the pseudocyst and the stomach. In order to visualize the perfect site for the anastomosis (away from vascular and biliary structures), IOUS is extremely useful and it is performed intragastric, guided by endoscopy [50] (fig 13).

The classic or laparoscopic approaches for small neuroendocrine tumor is often not possible without IOUS. In addition to the detection of the targeted lesions (most of the time unidentifiable by inspection and/or palpation), IOUS can establish the relation with the Wirsung duct

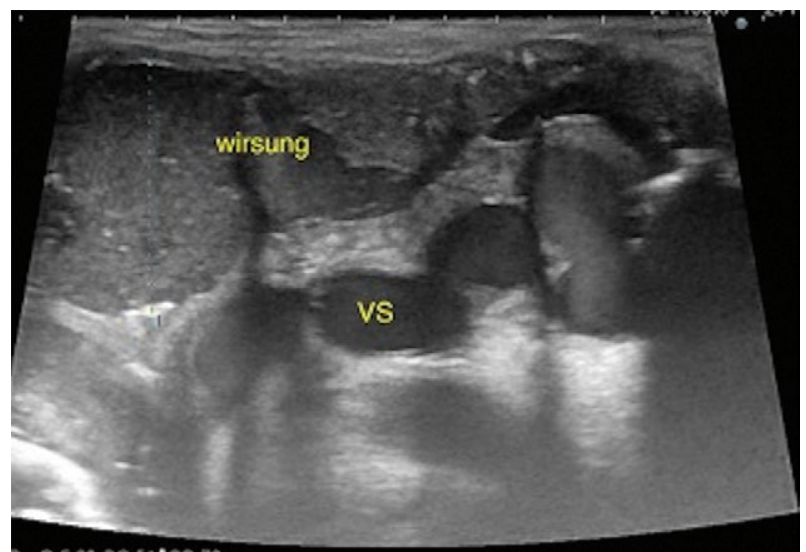

Fig 11. Chronic pancreatitis with the identification through IOUS (transversal section) of a very dilated Wirsung duct and a tortuous, dilated splenic vein (VS) (portal hypertension.

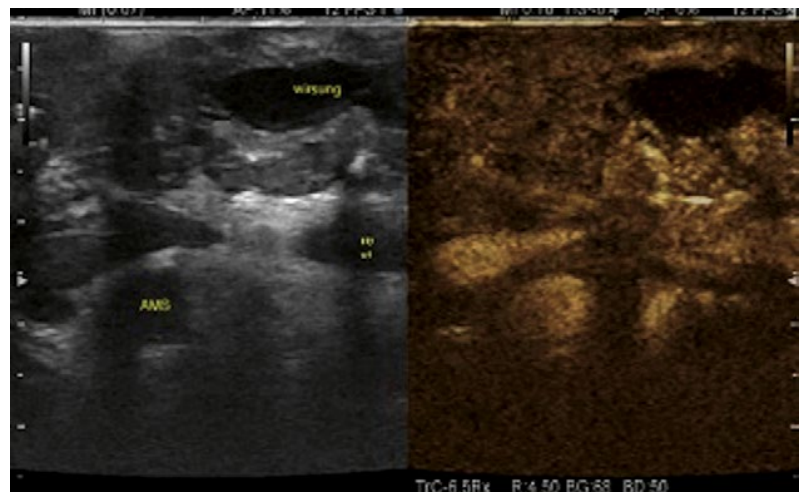

Fig 12. Chronic pseudotumoral pancreatitis (macroscopic intraoperative diagnosis). CE-IOUS (transversal section, SonoVue ${ }^{\circledR}$ ) indicates an inhomogeneous intake of the pancreatic parenchyma, without signs of malignancy ( $\mathrm{VS}=$ splenic vein; AMS = superior mesenteric artery).

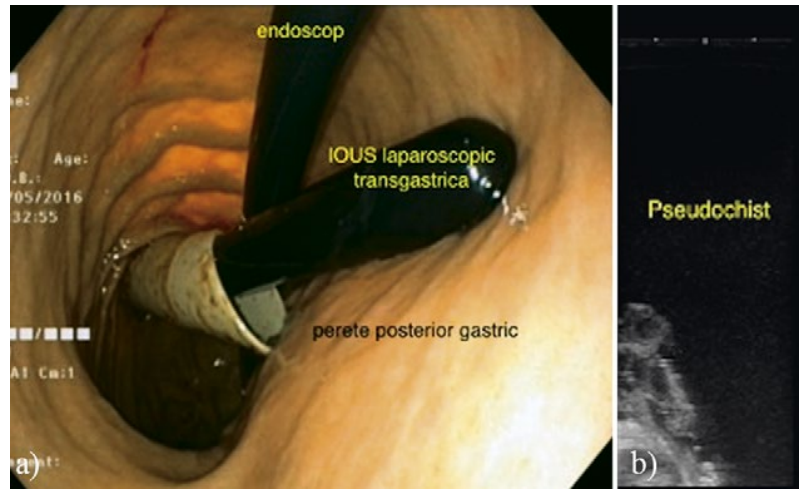

Fig 13. Laparoscopic intragastric (endoscopic assisted) IOUS prior to the drainage of a pancreatic pseudocyst located posterior from the stomach: a) intraoperative image with the laparoscopic transducer introduced into the stomach under endoscopic guidance; b) ultrasound image with the ideal site of anastomosis between the pseudocyst and the stomach $(*)$ (anechoic zone, without vascular or biliary structures) 


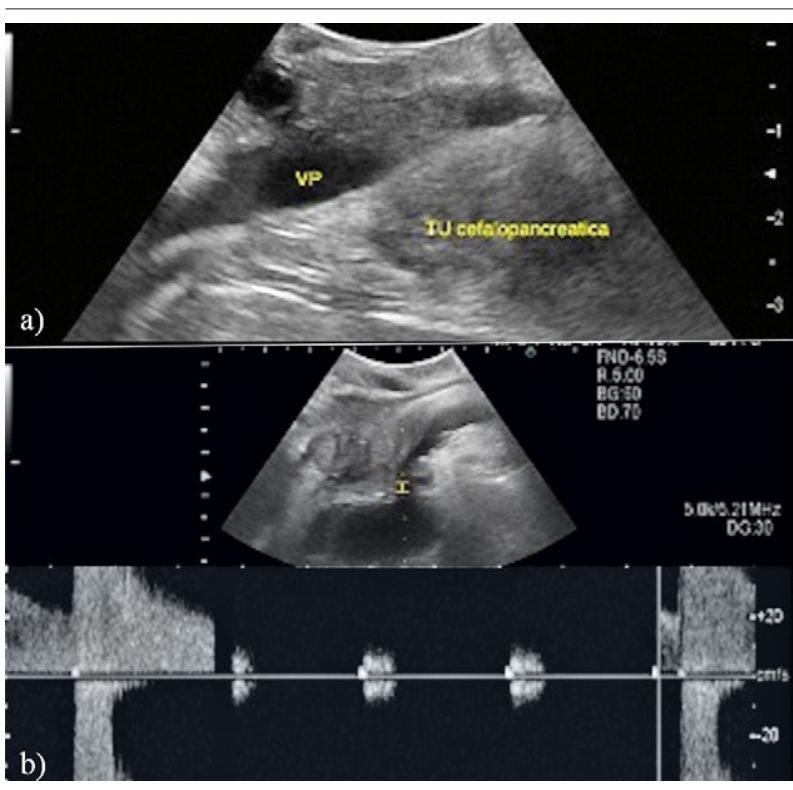

Fig 14. Locally advanced tumors of the head of the pancreas. IOUS aspects: a) with the invasion $(*)$ of the portomezenteric confluent; $b$ ) with the invasion of the superior mesenteric artery (AMS) $(\mathrm{Ao}=\mathrm{aorta}$; $\mathrm{TU}=$ tumor $)$ (pulsed Doppler) head, body or tail of the pancreas (fig 15) and for evaluating peripancreatic adenopathy (fig 16).

Pancreatic distal resections may be accompanied by the preservation or sacrifice of the spleen vessels. Depending on the indication, even if the spleen vessels (spleen artery) were ligated and sectioned, the spleen could be preserved and its vascularization is provided by gastric vessels and/or the gastro-epiploic arcade (Warshaw technique). The postoperative risk is represented by the splenic ischemia and the possible occurrence of the abscesses. The IOUS (Doppler color) and CE-IOUS assessment may reduce this risk by intraoperative selection of patients (fig 17).

Radiofrequency ablation of locally advanced pancreatic tumors is an ongoing technique in the dedicated centers of pancreas surgery [47,53-55]. In these selected cases, RFA produces tumor cytoreduction (by necrosis) in order to stabilize an aggressive pathology and to sustain an immune stimulation/modulation [56], in order to increase the survival rate [55]. Currently, endoscopic, percutaneous and surgical approaches have been described, all performed under ultrasound guidance [47,55,57] (fig 18).

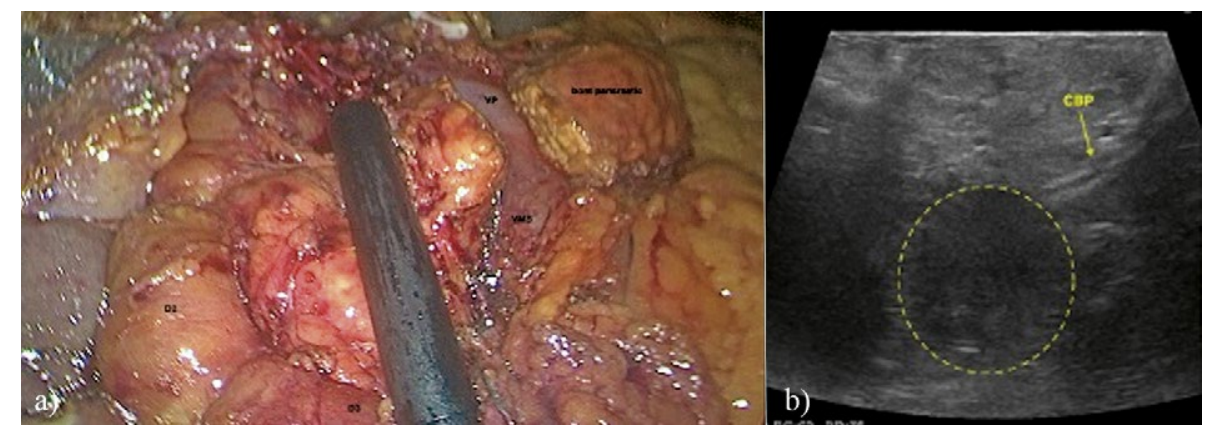

Fig 15. IOUS of malignant tumors of the head of the pancreas: a) intraoperative aspect with the laparoscopic transducer prior to performing a laparoscopic duodenopancreatectomy ( $\mathrm{VP}=$ portal vein; $\mathrm{VMS}=$ superior mesenteric vein; $\mathrm{D} 2 / \mathrm{D} 3=$ corresponding portion of the duodenum); b) laparoscopic IOUS of the tumor (TU) located distal from the common bile duct (CBP)

and the correct surgical conduct: enucleation or segmental resection [48].

The resectability of malignant pancreatic tumors might benefit in special cases from IOUS [51,52]. This technique might contribute to a rapid, non-invasive, non-dissection loco-regional evaluation, by identifying the criteria of unresectability: vascular invasions, lymph nodes or visceral metastases. The association of contrast-enhanced IOUS increases the sensitivity of the method by depicting the invasion of vascular structures. Pulsed Doppler ultrasonography analyzes the velocities before and after the specific vascular stenosis (fig 14).

In cases of laparoscopic treatment, IOUS is indispensable for the identification of tumors located in the

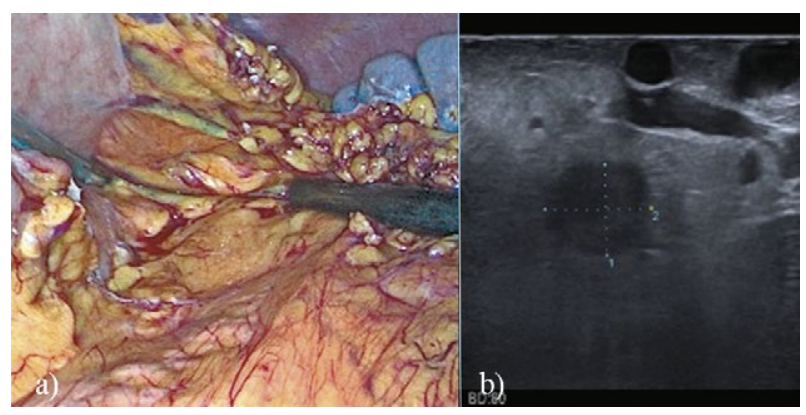

Fig 16. Laparoscopic IOUS of the tail of the pancreas: a) intraoperative aspect of using the laparoscopic transducer after the dissection of the body and tail of the pancreas and the spleen; b) ultrasound aspect, highlighting an adenopathy suspect for malignancy, situated posterior in the splenic hillum (VS = splenic vein; $\mathrm{AS}=$ splenic artery). 


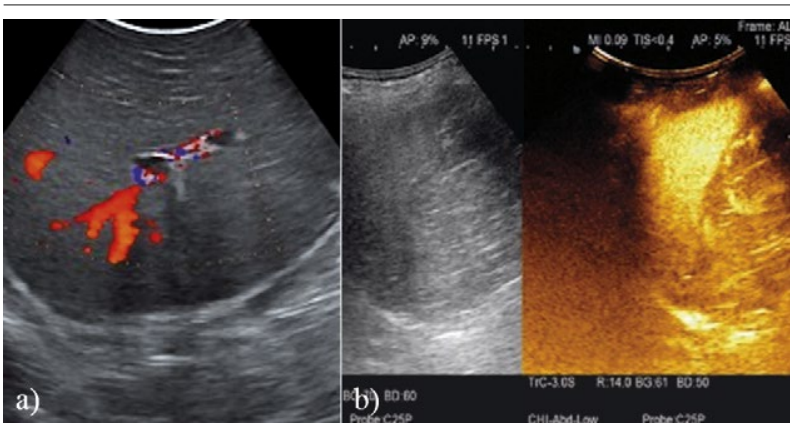

Fig 17. IOUS of the spleen, after the ligation of splenic vessels (artery and vein): a) IOUS aspect of the spleen, highlighting the arterial vascularization from short gastric vessels (color-Doppler ultrasound); b) transparietal CEUS aspect of the spleen (S), 30 days after the distal pancreatectomy, highlighting homogeneous vascular capture, without signs of ischemia.

Similar to intraoperative ablation techniques applied to hepatic tumors, the assessment of the post ablative necrosis area can be done through contrast-enhanced IOUS.

\section{Perspectives}

The continuous developing of technology and the completeness of the learning curves will result in a permanent development of the field of IOUS performed by surgeons. This technique adds a new ,3D feel” and has the potential to expand the indications of hepatic resections; more patients will benefit from radical surgery, preserving as much functional parenchyma as possible.

CE-IOUS has already a well-established role in the operating room, for diagnosis and treatment assessment efficacy. A big step forward can be achieved by improving the contrast agents, based on nanostructures and nanomedicine. The use of nanotechnology for the development of theranostic agents (with diagnostic and therapeutic properties) can bring researchers closer to the discovery of that „magic bullet” against cancer.

Elastography remains a rapid technique, easy to be performed intraoperatively, which might replace palpation especially for intraparenchymal tumors. It is a complementary technique to contrast-enhanced IOUS, with the potential to differentiate between benign and malignant tissues.

Intraoperative fluorescence and advanced navigation techniques in which preoperative imaging is directly projected in the surgical site (virtual reality) are already used in some centers of excellence worldwide. Even if the feasibility of these techniques is proved, the increased technical need and costs represent a major problem for implementing them on a large scale. Combining these techniques with IOUS brings clear benefits by $3 \mathrm{D}$ characterization of the already identified lesions.

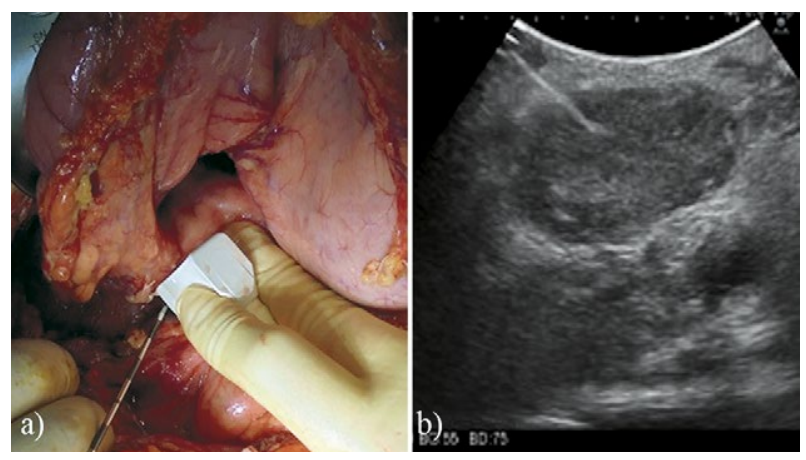

Fig 18. RFA of an unresectable, nonmetastatic tumor of the head of the pancreas - the ultrasound guided introduction of the RFA: a) intraoperative aspect; b) ultrasound aspect with the introduction of the needle used in order to perform the RFA (arrow) $(\mathrm{TU}=$ tumor; AMS = superior mesenteric artery).

\section{Conclusions}

The major advantages of IOUS indicated by literature and our clinical practice are represented by the correct diagnosis and staging of liver and pancreatic lesions, the guidance for surgery procedures (resection, ablative techniques) and the assessment of treatment efficacy in the same operative time. The need of a training specialized program for surgeons is mandatory.

\section{Conflict of interest: none}

\section{References}

1. Schlegel J, Diggdon P, Cuellar J. The use of ultrasound for localizing renal calculi. J Urol 1961;86:367-369.

2. Knight PR, Newell JA. Operative use of ultrasonics in cholelithiasis. Lancet 1963;1:1023-1025.

3. Sigel B, Coelho JC, Spigos DG, et al. Real-time ultrasonography during biliary surgery. Radiology 1980;137:531-533.

4. Fukuda M, Mima F, Nakano Y. Studies in echolaparoscopy. Scan J Gastroenterol 1982; 17(Suppl 78):186.

5. Jakimowicz JJ, Ruers TJM. Ultrasound-assisted laparoscopic cholecystectomy: preliminary experience. Dig Surg 1991;8:114-117.

6. Lane RJ, Coupland GA. Operative ultrasonic features of insulinomas. Am J Surg. 1982;144:585-587.

7. Shkolnik A, McLone DG. Intraoperative real-time ultrasonic guidance of ventricular shunt placement in infants. Radiology 1981;141:515-517.

8. Sigel B, Kraft AR, Nyhus LM, Coelho JC, Gavin MP, Spigos DG. Identification of a parathyroid adenoma by operative ultrasonography. Arch Surg 1981;116:234-235.

9. Eggemann H, Ignatov T, Beni A, Costa SD, Ortmann O, Ignatov A. Intraoperative Ultrasound in the Treatment of Breast Cancer. Geburtshilfe Frauenheilkd 2013;73:1028-1034.

10. Jakimowicz JJ. Intraoperative ultrasonography in open and laparoscopic abdominal surgery: an overview. Surg Endosc 2006;20 Suppl 2:S425-S435. 
11. Donadon M, Costa G, Torzilli G. State of the art of intraoperative ultrasound in liver surgery: Current use for staging and resection guidance. Ultraschall Med 2014;35:500-511.

12. Torzilli G, Procopio F, Fabbro D. Technological requirements for ultrasound-guided liver surgery. In: Torzilli G, editor. Ultrasound-Guided Liver Surgery. Italia: Springer; 2014:3-14.

13. Donadon M, Procopio F, Torzilli G. Tailoring the area of hepatic resection using inflow and outflow modulation. World J Gastroenterol 2013;19:1049-1055.

14. Torzilli G, Procopio F. State of the art of intraoperative ultrasound in liver surgery: Current use for resection-guidance. Chirurgia (Bucur) 2017;112:320-325.

15. Bartos A, Iancu I, Breazu C, Bartos D. Intraoperative U1trasound of the Liver: Actual Status and Indications. In: Rodrigo L. (ed). Liver Research and Clinical Management. 2018. doi:10.5772/intechopen.73856.

16. Torzilli G, Procopio F, Costa G. Resection guidance. In: Torzilli G. (Ed.). Ultrasound Guided Liver Surgery. Italia: Springer; 2014:117-168.

17. Xiang C, Liu Z, Dong J, Sano K, Makuuchi M. Precise anatomical resection of the ventral part of Segment VIII. Int J Surg Case Rep 2014;5:924-926.

18. Torzilli G, Procopio F, Palmisano A, et al. Total or partial anatomical resection of segment 8 using the ultrasoundguided finger compression technique. HPB (Oxford) 2011;13:586-591.

19. Torzilli G, Procopio F, Palmisano A, et al. New technique for defining the right anterior section intraoperatively using ultrasound-guided finger counter-compression. J Am Coll Surg 2009;209:e8-e11.

20. Torzilli G, Makuuchi M. Ultrasound-guided finger compression in liver subsegmentectomy for hepatocellular carcinoma. Surg Endosc 2004;18:136-139.

21. Torzilli G, Donadon M, Cimino M, Del Fabbro D, Procopio F, Botea F. Systematic subsegmentectomy by ultrasoundguided finger compression for hepatocellular carcinoma in cirrhosis. Ann Surg Oncol 2009;16:1843.

22. Kim HO, Kim SK, Son BH, et al. Intraoperative radiofrequency ablation with or without tumorectomy for hepatocellular carcinoma in locations difficult for a percutaneous approach. Hepatobiliary Pancreat Dis Int 2009;8:591596.

23. Machi J, Uchida S, Sumida K, et al. Ultrasound guided radiofrequency thermal ablation of liver tumors: Percutaneous, laparoscopic, and open surgical approaches. J Gastrointest Surg 2001;5:477-489.

24. Ishiko T, Beppu T, Sugiyama S, et al. Radiofrequency ablation with hand-assisted laparoscopic surgery for the treatment of hepatocellular carcinoma in the caudate lobe. Surg Laparosc Endosc Percutan Tech 2008;18:272-276.

25. El-Gendi A, El-Shafei M, Abdel-Aziz F, Bedewy E. Intraoperative ablation for small HCC not amenable for percutaneous radiofrequency ablation in Child A cirrhotic patients. J Gastrointest Surg 2013;17:712-718.

26. Parks K, Hagopian E. Introduction: The Importance of Ultrasound in a Surgical Practice. In: Hagopian E, Machi
J. (eds.). Abdominal Ultrasound for Surgeons. New York: Springer; 2014:3-6.

27. Eguchi A, Furuta T, Haraguchi M, Sugimachi K. Early stage hepatocellular carcinoma detected during intraoperative ultrasonography. Am J Gastroenterol 1994;89:595-598.

28. Kokudo N, Bandai Y, Imanishi H, et al. Management of new hepatic nodules detected by intraoperative ultrasonography during hepatic resection for hepatocellular carcinoma. Surgery 1996;119:634-640.

29. Gong NM, Yin HH, Cai WH, et al. IOUS and CE-IOUS during hepatic resection for patients with hepatocellular carcinoma in liver cirrhosis1. Clin Hemorheol Microcirc 2019;71:483-498.

30. Torzilli G. Contrast-enhanced intraoperative ultrasonography in surgery for liver tumors. Eur J Radiol 2004;51 Suppl:S25-29.

31. Pace C, Nardone V, Roma S, et al. Evaluation of ContrastEnhanced Intraoperative Ultrasound in the Detection and Management of Liver Lesions in Patients with Hepatocellular Carcinoma. J Oncol 2019,doi: 10.1155/2019/6089340.

32. Torzilli G, Olivari N, Moroni E, et al. Contrast-enhanced intraoperative ultrasonography in surgery for hepatocellular carcinoma in cirrhosis. Liver Transpl 2004;10(2 Suppl 1):S34-S38.

33. Sahani DV, Kalva SP, Tanabe KK, et al. Intraoperative US in patients undergoing surgery for liver neoplasms: comparison with MR imaging. Radiology 2004;232:810814.

34. Kulig J, Popiela T, Kłek S, et al. Intraoperative ultrasonography in detecting and assessment of colorectal liver metastases. Scand J Surg 2007;96:51-55.

35. Torzilli G. Does contrast-enhanced intraoperative ultrasonography impact radicality of hepatectomies for colorectal cancer liver metastases in spite of modern preoperative imaging? Analysis on a prospective cohort. Eur J Cancer 2008;6:16-23.

36. Torzilli G, Botea F, Procopio F. Use of contrast-enhanced intraoperative ultrasonography during liver surgery for colorectal cancer liver metastases - Its impact on operative outcome. Analysis of a prospective cohort study. Eur J Cancer Suppl 2008;6:16-23.

37. Claudon M, Dietrich CF, Choi BI, et al. Guidelines and good clinical practice recommendations for Contrast Enhanced Ultrasound (CEUS) in the liver - update 2012: A WFUMB-EFSUMB initiative in cooperation with representatives of AFSUMB, AIUM, ASUM, FLAUS and ICUS. Ultrasound Med Biol 2013;39:187-210.

38. Joo I. The role of intraoperative ultrasonography in the diagnosis and management of focal hepatic lesions. Ultrasonography 2015;34:246-257.

39. Ferrero A, Langella S, Russolillo N, Vigano L, Lo Tesoriere $\mathrm{R}$, Capussotti L. Intraoperative detection of disappearing colorectal liver metastases as a predictor of residual disease. J Gastrointest Surg 2012;16:806-814.

40. Omichi K, Inoue Y, Hasegawa K, et al. Differential diagnosis of liver tumours using intraoperative real-time tissue elastography. Br J Surg 2015;102:246-253. 
41. Inoue $\mathrm{Y}$, Takahashi M, Arita J, et al. Intra-operative freehand real-time elastography for small focal liver lesions: "visual palpation" for non-palpable tumors. Surgery 2010;148:1000-1011.

42. Moris D, Tsilimigras DI, Kostakis ID, et al. Anatomic versus non-anatomic resection for hepatocellular carcinoma: A systematic review and meta-analysis. Eur J Surg Oncol 2018;44:927-938.

43. Torzilli G, Montorsi M, Donadon M, et al. "Radical but conservative" is the main goal for ultrasonography-guided liver resection: prospective validation of this approach. $\mathrm{J}$ Am Coll Surg 2005;201:517-528.

44. NCCN. Clinical Practice Guidelines in Oncology Hepatobiliary Cancers Version 3. 2018 Available from: https:// www.nccn.org/professionals/physician_gls/default.aspx

45. Eun HS, Lee BS, Kwon IS, et al. Advantages of Laparoscopic Radiofrequency Ablation Over Percutaneous Radiofrequency Ablation in Hepatocellular Carcinoma. Dig Dis Sci 2017;62:2586-2600.

46. Wong J, Lee KF, Yu SC, et al. Percutaneous radiofrequency ablation versus surgical radiofrequency ablation for malignant liver tumours: the long-term results. HPB (Oxford) 2013;15:595-601.

47. Bartos A, Breazu C, Sparchez Z, Tantau M, Iancu C, Bartos D. Radiofrequency ablation of locally advanced pancreatic tumors. The surgical approach. J Gastrointest Liv Dis 2018;27:102-104.

48. D’Onofrio M, Vecchiato F, Faccioli N, Falconi M, Pozzi Mucelli R. Ultrasonography of the pancreas. Abdom Imaging 2007;32:200-206.

49. Simo KA, Niemeyer DJ, Swan RZ, Sindram D, Martinie JB, Iannitti DA. Laparoscopic transgastric endolumenal cystogastrostomy and pancreatic debridement. Surgical endoscopy 2014;28:1465-1472.

50. Bartos D, Bartos A. Endoscopy-assisted, single transgastric trocar, laparoscopic pseudocysto-gastrostomy. A minimally invasive alternative for drainage of large pseudocysts. Ann Ital Chir 2019 May 21;8:S0003469X19029 415.

51. Oncology NCPGi. Pancreatic Adenocarcinoma 2018 Available from: https://www.nccn.org/professionals/physician gls/pdf/pancreatic.pdf.

52. Lin H, Ma Y, Wang JZ, et al. Analysis of 300 consecutive cases of pancreatic adenocarcinoma in a single-center in China. Hepatobiliary Pancreat Dis Int 2016;15:189197.

53. Paiella S, Malleo G, Cataldo I, et al. Radiofrequency ablation for locally advanced pancreatic cancer: SMAD4 analysis segregates a responsive subgroup of patients. Langenbecks Arch Surg 2018;403:213-220.

54. Paiella S, Salvia R, Ramera M, et al. Local Ablative Strategies for Ductal Pancreatic Cancer (Radiofrequency Ablation, Irreversible Electroporation): A Review. Gastroenterol Res Pract 2016;2016:4508376.

55. Linecker M, Pfammatter T, Kambakamba P, DeOliveira ML. Ablation Strategies for Locally Advanced Pancreatic Cancer. Dig Surg 2016;33:351-359.

56. Chu KF, Dupuy DE. Thermal ablation of tumours: biological mechanisms and advances in therapy. Nat Rev Cancer 2014;14:199-208.

57. D’Onofrio M, Crosara S, De Robertis R, et al. Percutaneous Radiofrequency Ablation of Unresectable Locally Advanced Pancreatic Cancer: Preliminary Results. Technol Cancer Res Treat 2017;16:285-294. 